\title{
NICA-MPD: azimuthal and femtoscopic particle correlations
}

\author{
V.A. Okorokov a \\ National Research Nuclear University MEPhI (Moscow Engineering Physics Institute), \\ Kashirskoe Shosse 31, 115409 Moscow, Russian Federation
}

Received: 27 September 2015

\begin{abstract}
The discussion is focused on the study of the fundamental symmetries $(\mathcal{P} / \mathcal{C P})$ of $Q C D$ and geometry of the particle source. The combination of correlators corresponding to the absolute asymmetry of distribution of electrically charged particles with respect to the reaction plane in heavy ion collisions is studied. A significant decrease of the absolute asymmetry is observed in the intermediate energy range which can be considered as indication of possible transition to predominance of hadronic states over quarkgluon degrees of freedom in the mixed phase created in heavy ion collisions at intermediate energies. For the investigation of the energy evolution of the geometric properties of the particle source the use of femtoscopic radii scaled on the averaged radius of colliding ions is suggested. This approach allows the expansion of the set of interaction types, in particular, on the collisions of non-symmetrical ion beams which can be studied within the framework of common treatment. There is no sharp changing of femtoscopic parameter values with increasing of the initial energy. The suggestions are made for future advancement of these studies on NICA-MPD.
\end{abstract}

PACS. 25.75.-q Relativistic heavy-ion collisions, 25.75.Gz Particle correlations and fluctuations

\section{Introduction}

There is a fundamental interrelation between geometry and fundamental properties of QCD Lagrangian. The vacuum of QCD is a very complicated matter with rich structure, which can corresponds to the fractal-like geometry 11. Of particular interest in the studies of nucleus-nucleus collisions is a possibility to create a new state of strongly interacting matter where some of the fundamental symmetries may be violated. The non-trivial topology of QCD vacuum opens the possibility for existence of metastable domains which possess of various properties with respect to the discrete $\mathcal{P} / \mathcal{C P}$ symmetries. According to predictions of the theory at finite temperature 2 decays of such domains or classical transitions (sphalerons) between them in deconfinement phase of color charges with restored chiral symmetry can result in local topologically induced violation of $\mathcal{P} / \mathcal{C P}$ invariance in strong interactions $-l \mathcal{T} \mathcal{I} \mathcal{P}$ effect. In the presence of background Abelian electromagnetic field the $l \mathcal{T} \mathcal{I P}$ effect can leads to the separation of secondary charged particles toward the magnetic field 3,4. This phenomenon called also chiral magnetic effect (CME) is the experimental manifestation of the local topologically induced $\mathcal{P} / \mathcal{C P}$ parity violation in strong interactions. On the other hand, at present femtoscopic measurements in particular that based on Bose-Einstein correlations are a unique experimental method for the determination of the sizes and lifetimes of the sources, i.e. $4 \mathrm{D}$

\footnotetext{
a e-mail: VAOkorokov@mephi.ru; Okorokov@bnl.gov
}

geometry, in high energy and nuclear physics. The study of nucleus-nucleus $(A+A)$ collisions in wide energy domain by correlation femtoscopy seems important for better understanding both the equation of state (EOS) of strongly interacting matter and the general dynamic features of soft processes. Therefore, the future experimental studies of the non-trivial structure of the QCD vacuum and geometry of particle source in the NICA-MPD energy domain allow an important progress with respect to the fundamental symmetries and non-perturbative properties of strong interactions.

\section{Fundamental symmetries of QCD at finite temperature}

With taking into account possible local strong $\mathcal{P} / \mathcal{C P}$ violation the invariant distribution of final state particles with certain sign of electric charge $\alpha(\alpha=+,-)$ can be written as follows:

$$
E \frac{d^{3} N_{\alpha}}{d \boldsymbol{p}}=\frac{1}{2 \pi} \frac{d^{2} N_{\alpha}}{p_{\perp} d p_{\perp} d y}\left[1+2 \sum_{n=1}^{\infty} \sum_{m=1}^{2} k_{n, \alpha}^{m} F^{m}(n \Delta \phi)\right] .
$$

Here $\Delta \phi \equiv \phi-\Psi_{R P}, \phi$ is the azimuthal angle of the particle under study, $\Psi_{R P}$ the azimuthal angle of the reaction plane, $F^{1,2}(x) \equiv \cos (x), \sin (x), k_{n, \alpha}^{1} \equiv v_{n, \alpha}$ is the collective flow of the $n$-th order, the parameters $k_{n, \alpha}^{2} \equiv a_{n, \alpha}$ describe the effect of $\mathcal{P} / \mathcal{C P}$ violation. It should be emphasized that the CME is the collective effect and its 
investigation is possible via correlation analysis only because $\left\langle a_{n, \alpha}\right\rangle=0$ if the one-particle distributions are averaged over the event sample. According to the theory, the correlator which contains only the contribution of possible $\mathcal{P} / \mathcal{C P}$ violation effect is given by equation $\left\langle\mathbf{K}_{n, \alpha \beta}^{T}\right\rangle=$ $\left\langle a_{n, \alpha} a_{n, \beta}\right\rangle$, where $\alpha, \beta$ are the electric charge signs of secondary particles. Only the first harmonic coefficient is analyzed so far because as expected $a_{1, \alpha}$ accounts for most of the effect under study. Thus the following notation $\left\langle\mathbf{K}_{1, \alpha \beta}^{T}\right\rangle \equiv\left\langle\mathbf{K}_{\alpha \beta}^{T}\right\rangle$ is used below. The structure of $\left\langle\mathbf{K}_{\alpha \beta}^{T}\right\rangle$ is described in detail elsewhere [3, 5]. The experimental correlator proposed in [6] is defined as follows: $\left\langle\mathbf{K}_{\alpha \beta}^{E}\right\rangle=$ $\left\langle\cos \left(\phi_{\alpha}+\phi_{\beta}-2 \Psi_{R P}\right)\right\rangle$. This observable is sensitive to the effect of possible local strong $\mathcal{P} / \mathcal{C P}$ violation and measures the charge separation with respect to the reaction plane. Both theoretical and experimental correlators are averaged over pairs of particles under study in the event and over all events in the sample. It should be mentioned that the correlators defined above are $\mathcal{P}$-even quantities therefore $\left\langle\mathbf{K}_{\alpha \beta}^{E}\right\rangle$ may contain contributions from background effects unrelated to possible local strong $\mathcal{P} / \mathcal{C P}$ violation. Theoretical and experimental correlators are related by the following equation:

$$
\left\langle\mathbf{K}_{\alpha \beta}^{E}\right\rangle=\tilde{B}-\left\langle\mathbf{K}_{\alpha \beta}^{T}\right\rangle,
$$

where $\tilde{B}$ is the total background contribution discussed in [5]. The absolute asymmetry with respect to the reaction plane for the azimuthal distribution of the electric charges in final state is define as follows [5]:

$$
A_{a}=-\left[\left\langle\mathbf{K}_{ \pm \pm}^{E}\right\rangle-\left\langle\mathbf{K}_{ \pm \mp}^{E}\right\rangle\right] .
$$

In the framework of CME model [3] for the ideal case of chiral limit and an extremely large magnetic field the following relations have been derived [5]

$$
\left\langle Q^{2}\right\rangle \propto \Gamma_{C S}, \quad A_{a} \propto \Gamma_{C S} .
$$

Here $\left\langle Q^{2}\right\rangle$ is the average square of the charge difference (in units of $e$ ) between opposite sides of the reaction plane, $\Gamma_{C S}$ is the classical transition rate, i.e. the Chern-Simons diffusion rate. It should be noted that the separation of a possible CME signal from background effects is an important and difficult task for $\left\langle\mathbf{K}_{\alpha \beta}^{E}\right\rangle$ and consequently for quantity $A_{a}$. Based on the available experimental results and its interpretation for wide initial energy range one can assume at qualitative level only that the sizable contribution in $A_{a}$ will be due to correlations driven by local $\mathcal{P} / \mathcal{C P}$ violation in strong interactions [5].

Fig. 1 shows the energy dependence of $A_{a}$ for semicentral events in various bins of centrality for heavy ion beams. The equation for the Chern-Simons diffusion rate in the case of finite $B$ had been derived in [7. The background magnetic field typically created in relativistic heavy ion collisions is characterized by the strength $B \sim T^{2}[3$, 8, 9. The regime of finite external magnetic field is investigated in the paper and it is observed that the curves for $B=0$ and for the case $B=T^{2}$ coincide completely for all centrality bins under study, i.e. for the full centrality domain 20-60\% shown in Fig. 11. But relations in (3) have been obtained for extremely strong magnetic field. Thus the energy dependence for Chern-Simons diffusion rate is computed for external Abelian magnetic field with various strengths as follows [7]:

$$
\Gamma_{C S}^{B \neq 0}=\Gamma_{C S}^{B=0}\left[1+\zeta^{2} /\left(6 \pi^{4}\right)\right], \quad \zeta \equiv B / T^{2} .
$$

It should be noted that at fixed $\zeta$ the functional dependence of $\Gamma_{C S}$ on $T$ is the same both for $B=0$ and for the general case of the presence of a finite external Abelian magnetic field. The function suggested in [10] for the description of the energy dependence of the chemical freezeout temperature agrees with available experimental data quite reasonably for energies up to $\sqrt{s_{N N}}=200 \mathrm{GeV}$ 11 and for all centralities 12 under study. Therefore the analytic dependence $T\left(\sqrt{s_{N N}}\right)$ from [10] is used for estimations of $\Gamma_{C S}$ in the energy domain under study. The smooth curves in Fig. 11 are the energy dependencies of the Chern-Simons diffusion rate in the strong coupling regime at $B=0$ (solid), $B=5 T^{2}$ (dashed) and $B=10 T^{2}$ (dotted). The norm for solid curves is the STAR point for $\mathrm{Au}+\mathrm{Au}$ at $\sqrt{s_{N N}}=200 \mathrm{GeV}$. The shaded bands for the Chern-Simons diffusion rate at $B=0$ are defined by uncertainties of $T$ value at fixed initial energy due to errors of parameters in the analytical function describing of $T\left(\sqrt{s_{N N}}\right)$ experimental dependence [10]. As seen, the phenomenological curves $\Gamma_{C S}\left(\sqrt{s_{N N}}\right)$ are in an area caused by the spread of $T$ values at fixed $\sqrt{s_{N N}}$ for following wide range of changing of strength of external Abelian magnetic field $B \leq 5 T^{2}$ at any initial energies under study, and also at $\sqrt{s_{N N}} \lesssim 12 \mathrm{GeV}$ for all the range of $B$ considered in Fig. 1, The functional behavior of $\Gamma_{C S}\left(\sqrt{s_{N N}}\right)$ does not depend on $B$ in high energy domain at changing of $B$ within the range from $B=0$ until at least the value $B=10 T^{2}$ (Fig. 1).

One sees the some decreasing of $A_{a}$ for LHC energy as compared to initial energy domain $\sqrt{s_{N N}} \sim 100 \mathrm{GeV}$, the absolute value of decreasing increases for more peripheral events. The parameter $A_{a}$ goes down significantly with $\sqrt{s_{N N}}$ decreasing for the intermediate initial energy domain $7.7-20 \mathrm{GeV}$ in semicentral heavy ion collisions (Fig. 1). For each centrality bin under study the estimations for $A_{a}\left(\sqrt{s_{N N}}\right)$ correspond to the energy dependence of $\Gamma_{C S}$ at $B=0$ on the qualitative level with some enhancement of experimental points over phenomenological curves in the range $\sqrt{s_{N N}} \sim 20-40 \mathrm{GeV}$ for events with $20-30 \%$ (Fig. 1a) and 30-40\% (Fig. 1b) centrality. Perhaps, for better description of $A_{a}\left(\sqrt{s_{N N}}\right)$ at $\sqrt{s_{N N}} \sim 20-40 \mathrm{GeV}$ the influence of external $B$ on $\Gamma_{C S}$ should be taken into account. This suggestion agrees with the dependence of $B$ on initial energy [8] which demonstrates that $B$ in the intermediate energy range $\sqrt{s_{N N}} \sim 20-40 \mathrm{GeV}$ reaches very large values with respect to the strength of $B$ at $\sqrt{s_{N N}} \sim 100 \mathrm{GeV}$. A qualitative agreement is observed between values of the $A_{a}$ parameter calculated for $\mathrm{Au}+\mathrm{Au}$ collisions and phenomenological curves for any strength values of $B$ under study. This feature can be considered as some evidence of validity of relations in (3) derived at the ultimate strong external Abelian magnetic field for 


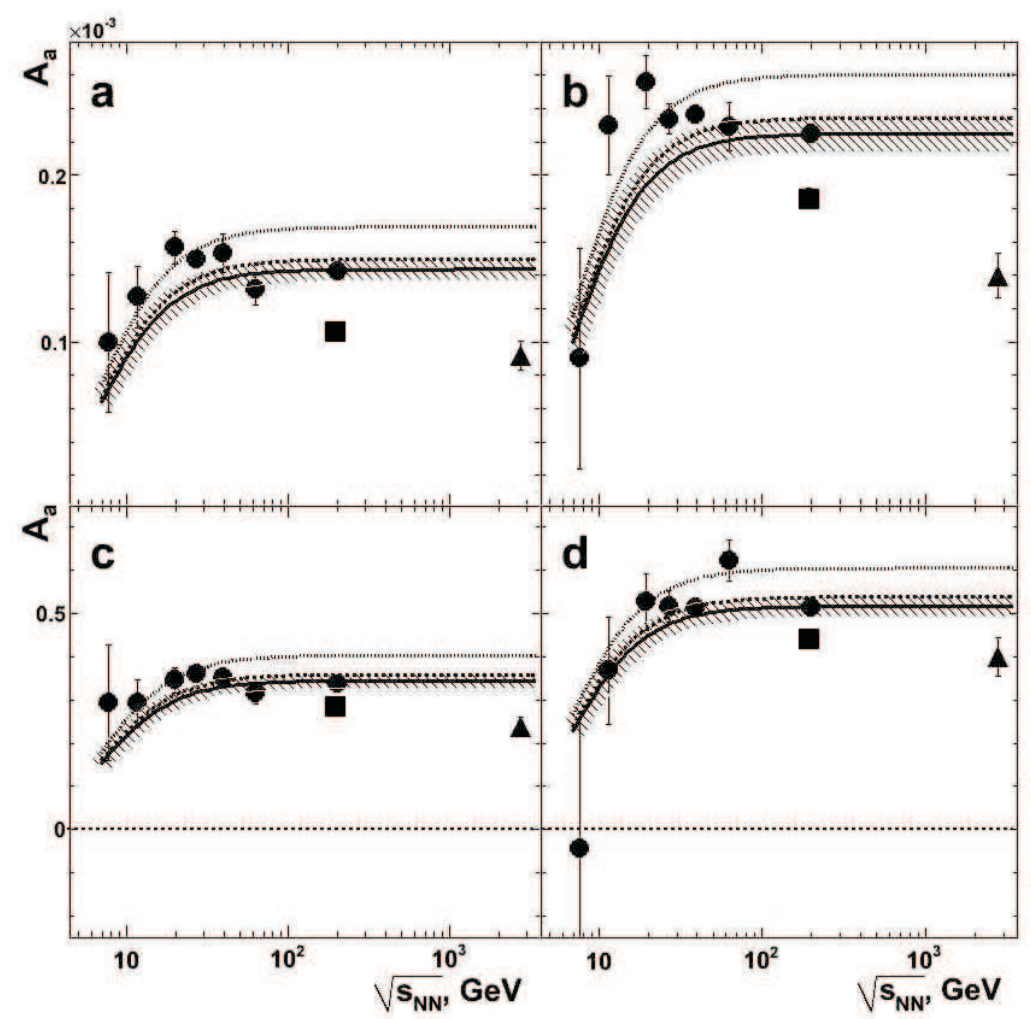

Fig. 1. The energy dependence of $A_{a}$ for heavy ion collisions in various centrality bins: a $-20-30 \%, \mathrm{~b}-30-40 \%, \mathrm{c}-40-50 \%$ and $\mathrm{d}-50-60 \%$ 5. Experimental points are shown as follows: $\bullet-\mathrm{Au}+\mathrm{Au}, \boldsymbol{\Delta}-\mathrm{Pb}+\mathrm{Pb}$ and $\boldsymbol{\square}-\mathrm{U}+\mathrm{U}$. Smooth curves correspond to the energy dependence of the Chern-Simons diffusion rate in the strong coupling regime for external magnetic field with strength $B=0$ (solid), $B=5 T^{2}$ (dashed) and $B=10 T^{2}$ (dotted). The shaded areas for $\Gamma_{C S}$ at $B=0$ are defined by uncertainties of the $T$ value at fixed initial energy due to errors of the parameters in the analytic function describing of the experimental dependence $T\left(\sqrt{s_{N N}}\right)$.

heavy ion collisions. The decrease of $\Gamma_{C S}\left(\sqrt{s_{N N}}\right)$ observed at $\sqrt{s_{N N}}<20 \mathrm{GeV}$ should lead to attenuation of $\mathrm{CME}$ and its manifestation on experiment at intermediate energies. Taking into account conditions which are essential for $\mathrm{CME}$ one can suppose the following hypothesis. The change of behavior of the $A_{a}\left(\sqrt{s_{N N}}\right)$ dependence observed at the transition from the high energy domain down to the intermediate energy range may be driven by the predominance of hadronic colorless states over the quark-gluon deconfinement phase at $\sqrt{s_{N N}}<19.6 \mathrm{GeV}$ and, as a consequence, by the decrease of CME. Thus the behavior of the experimental quantity $A_{a}$ vs collision energy agrees with qualitative expectation for transition to the predominance of the hadronic phase in the domain $\sqrt{s_{N N}}<19.6$ $\mathrm{GeV}$ and with the decreasing of the Chern-Simons diffusion rate at intermediate initial energies.

\section{Femtoscopic correlations}

The discussion below is focused on the specific case of femtoscopy, namely, on correlations in pairs of identical charged pions with small relative momenta - HBT-interferometry - in $A+A$ collisions. The set of main femtoscopic observables $\mathcal{G} \equiv\left\{\mathcal{G}_{1}^{i}\right\}_{i=1}^{4}=\left\{\lambda, R_{\mathrm{s}}, R_{\mathrm{o}}, R_{1}\right\}$ is only under consideration here. $\mathcal{G}$ characterizes the correlation strength and source's 4-dimensional geometry at the freeze-out stage completely, some important additional observables which can be calculated with help of HBT radii are discussed elsewhere 13, 14. The most central collisions are usually used for studying the space-time characteristics of final-state matter, in particular, for the discussion of the global energy dependence of femtoscopic observables. Therefore the scaled parameters $\mathcal{G}^{i}, i=2-4$ are calculated as follows 13 :

$$
R_{i}^{n}=R_{i} / R_{A}, i=s, o, l .
$$

Here $R_{A}=R_{0} A^{1 / 3}$ is the radius of the spherically-symmetric nucleus, $R_{0}=(1.25 \pm 0.05) \mathrm{fm}$ [15]. The change $R_{A} \rightarrow$ $\left\langle R_{A}\right\rangle=0.5\left(R_{A_{1}}+R_{A_{2}}\right)$ is made in relation (4) in the case of non-symmetric nuclear collisions 13 . In the general case the scale factor in (4) should take into account the centrality of nucleus-nucleus collisions. The normalization procedure suggested in 13 allows the general consider- 


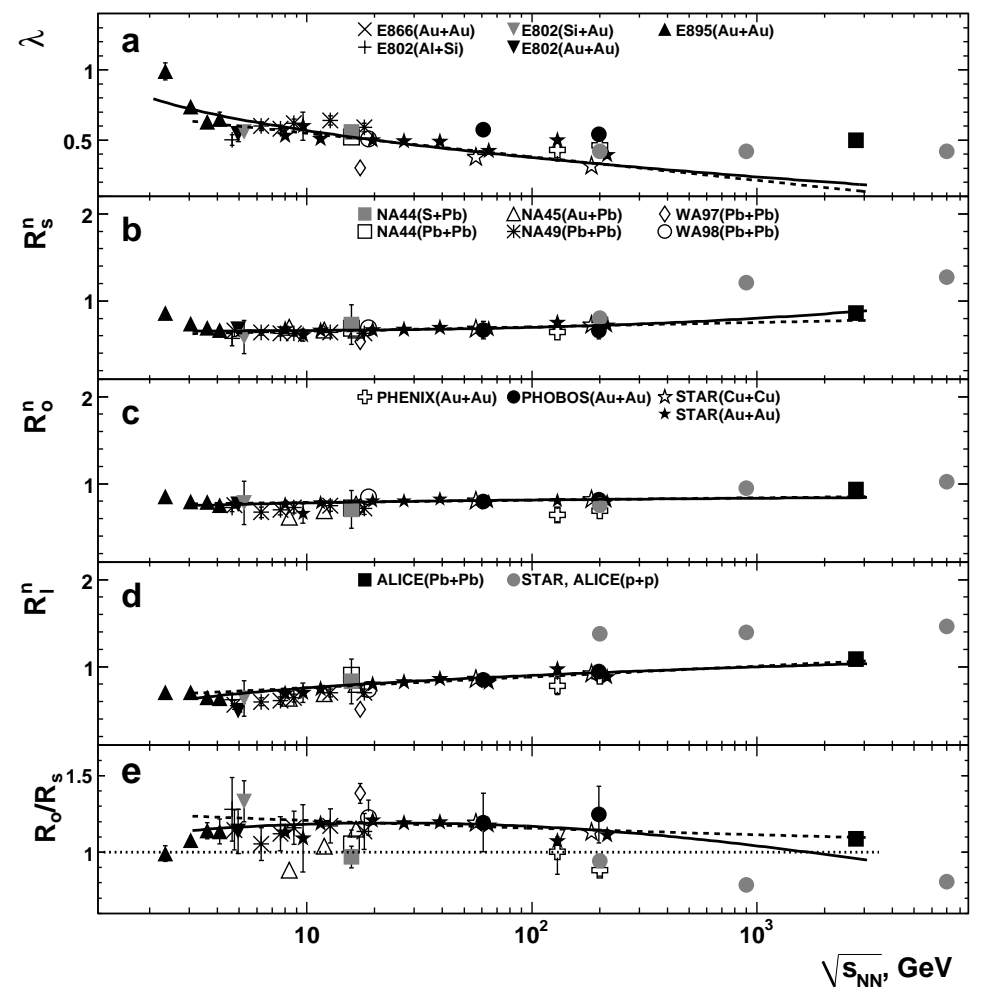

Fig. 2. The energy dependence of the $\lambda$ parameter (a), the scaled HBT-radii $(\mathrm{b}-\mathrm{d})$ and the ratio $R_{\mathrm{o}} / R_{\mathrm{S}}(\mathrm{e})$ in various collisions. Experimental data are from [14. Statistical errors are shown (for NA44 - total uncertainties). The solid lines in (a d) correspond to the fits by function (5) and the dashed lines - to the fits by the specific case of (5) at fixed $a_{3}=1.0$. Smooth solid and dashed curves in (e) correspond to the ratio $R_{\mathrm{o}} / R_{\mathrm{S}}$ calculated from the fit results for $R_{\mathrm{s}}^{n}$ and $R_{\mathrm{o}}^{n}$ in $A+A$, the dotted line is the level $R_{\mathrm{o}} / R_{\mathrm{S}}=1$.

ation of all available data for nucleus-nucleus collisions together with the proton-proton $(p p)$ results at high energies with replacing $R_{A} \rightarrow R_{p}$ in (4). A detailed study for (quasi)symmetric heavy ion collisions 13 demonstrates that the fit function $\left(\varepsilon \equiv s_{N N} / s_{0}, s_{0}=1 \mathrm{GeV}^{2}\right)$

$$
f\left(\sqrt{s_{N N}}\right)=a_{1}\left[1+a_{2}(\ln \varepsilon)^{a_{3}}\right]
$$

agrees reasonably with experimental $\mathcal{G}^{i}\left(\sqrt{s_{N N}}\right), i=1-4$ at any collision energy for $\lambda$ and at $\sqrt{s_{N N}} \geq 5 \mathrm{GeV}$ for HBT radii. Fig. 2 shows the energy dependence of $\lambda$ (a), the scaled HBT-radii $(\mathrm{b}-\mathrm{d})$ and the $R_{o} / R_{s}$ ratio (e) for both the symmetric and non-symmetric collisions of various nuclei. Fits of experimental dependencies for $A+A$ interactions are made by (5) in the same energy domains as well as for (quasi)symmetric heavy ion collisions. Fit curves are shown in Fig. 2 by solid lines for (5) and by dashed lines for the specific case of fit function at $a_{3}=1.0$ with taking into account statistical errors. The fit by (5) underestimates the $\lambda$ value at the LHC energy $\sqrt{s_{N N}}=2.76 \mathrm{TeV}$ significantly. The $\lambda$ values for asymmetric nucleus-nucleus collisions at intermediate energies $\sqrt{s_{N N}} \lesssim 20 \mathrm{GeV}$ agree well with values of $\lambda$ in symmetric heavy ion collisions at close energies. On the other hand the development of some approach is required in order to account for the type of colliding beams in the case of $\lambda$ parameter and improve the quality of approximation. Smooth curves for the normalized HBT radii and the ratio $R_{o} / R_{s}$ are in reasonable agreement with experimental dependencies in the fitted domain of collision energies $\sqrt{s_{N N}} \geq 5 \mathrm{GeV}$ (Figs. 2b - e). The scaled HBT-radii in $p p$ are significantly larger than those in $A+A$ collisions at close energies. Because of the feature of Regge theory [16] the following relation is suggested to take into account the expansion of proton with energy: $R_{p}=r_{0}\left(1+k \sqrt{\alpha_{\mathcal{P}}^{\prime} \ln \varepsilon}\right)$, where $r_{0}=(0.877 \pm 0.005) \mathrm{fm}$ is the proton's charge radius [17, parameter $\alpha_{\mathcal{P}}^{\prime} \propto \ln \varepsilon$ because the diffraction cone shrinkage speeds up with collision energy in elastic $p p$ scattering [18]. $k$ is defined by the boundary condition $R_{p} \rightarrow 1 / m_{\pi}$ at $\varepsilon \rightarrow \infty$ with appropriate choice of asymptotic energy $\sqrt{s_{N N}^{a}}$. A detailed study demonstrates that the increasing of $\sqrt{s_{N N}^{a}}$ from $6 \mathrm{PeV}\left[19\right.$ to $10^{3} \mathrm{PeV}$ influences weakly $R_{i}^{n}, i=s, o, l$ in $p p$ collisions and calculations are made for the first case. The normalized transverse radii agree in both the $p p$ and the $A+A$ collisions (Figs. $2 \mathrm{~b}$, c) at $\sqrt{s_{N N}}=200 \mathrm{GeV}$ with an excess of $R_{s}^{n}$ in $p p$ with respect to $A+A$ in the $\mathrm{TeV}$-region. The $R_{l}^{n}$ in $p p$ is larger 
than that for $A+A$ in the domain $\sqrt{s_{N N}} \geq 200 \mathrm{GeV}$. It should be stressed that additional study is important, at least, for the choice of $R_{p}(\varepsilon)$.

\section{Future investigations}

It should be noted that the requirements for main tracker of MPD - time-projection chamber - give the possibility for measurements of various correlations, in particular discussed above, with high quality and statistic.

The study at NICA-MPD with heavy ion beams allows the precise measurement of the $A_{a}\left(\sqrt{s_{N N}}\right)$ dependence in the energy region of sharp decrease of the absolute asymmetry. These future investigations will be useful for testing the hypothesis about the attenuation of the CME due to the transition to the predominance of the hadronic phase in the domain $\sqrt{s_{N N}}<19.6 \mathrm{GeV}$. Furthermore in this paper it is suggested that at finite temperature $(T)$ the transition rate between vacuum states with different $N_{C S}$ is dominated by the transition rate due to sphalerons only, without consideration of the exponentially suppressed transitions due to instantons. But it should be mentioned that some additional study and justification may be required for this approach, especially in the temperature range $T_{c}<T<3 T_{c}$ [20, where $T_{c}$ is the temperature of phase transition to the deconfinement state of color charges. Such investigations can be proposed for the NICA-MPD physics program.

New experimental data are important for the verification of the suggestion of separate dependencies $\lambda\left(\sqrt{s_{N N}}\right)$ for moderate and heavy ion collisions. The energy dependence is almost flat for the scaled difference $\delta^{n} \equiv\left(R_{o}^{2}-\right.$ $\left.R_{s}^{2}\right) R_{A}^{-2}$ in $A+A$ collisions within large error bars [13, 14 . The indication of a possible curve knee at $\sqrt{s_{N N}} \sim 10-20$ $\mathrm{GeV}$ obtained in the STAR high-statistics data agrees with other results in the framework of the phase-I of the BES program at RHIC. But additional precise measurements at NICA-MPD with various beams and $\sqrt{s_{N N}}$ may be crucially important in order to confirm this feature in the energy dependence of the important parameter $\delta^{n}$. Furthermore there is a wide set of femtoscopic measurements available at NICA-MPD with both the identical heavier particles and the non-identical particle pairs. The $\Lambda \Lambda$ correlations can be used for the search for exotic states in QCD, for instance, $H$ dibaryons 21]. The non-identical particle correlations with kaons allow us to obtain information about the space-time asymmetry of particle emission, the final state interaction and some exotic objects like kaonic atoms.

\section{Summary}

The absolute asymmetry is introduced in order to investigate the evolution of CME in heavy ion collisions with initial energy. Dependence on $\sqrt{s_{N N}}$ has been obtained for $A_{a}$ based on the experimental correlators. The energy dependence of absolute asymmetry for semicentral events in heavy ion collisions shows sharp decreasing at $\sqrt{s_{N N}}<19.6 \mathrm{GeV}$, almost constant behavior up to $\sqrt{s_{N N}} \simeq 200 \mathrm{GeV}$ with some decreasing with further energy increasing. $A_{a}\left(\sqrt{s_{N N}}\right)$ dependence qualitatively corresponds to the energy dependence of the Chern-Simons diffusion rate. $A_{a}\left(\sqrt{s_{N N}}\right)$ indicates possibly the onset of predominance of the hadronic states over the phase of color degrees of freedom in the deconfinement state in the matter created in heavy ion collisions with initial energies in the domain $\sqrt{s_{N N}} \lesssim 11.5-19.6 \mathrm{GeV}$. The energy dependence is investigated for the main femtoscopic parameters deduced in the framework of the Gauss approach. There is no dramatic change of femtoscopic parameter values in $A+A$ with increasing $\sqrt{s_{N N}}$ in the domain of collision energies $\sqrt{s_{N N}} \geq 5 \mathrm{GeV}$. Some normalized HBT radii in $p p$ are significantly larger than those in $A+A$ collisions especially in the $\mathrm{TeV}$-region. The fit curves demonstrate qualitative agreement with experimental $A+A$ data for $\lambda$ at all available collision energies and for normalized HBT radii in the energy domain $\sqrt{s_{N N}} \geq 5 \mathrm{GeV}$.

The investigations of azimuthal and femtoscopic correlations at intermediate energies with high statistics at NICA-MPD can provide new important information for better understanding the structure of the $\mathrm{QCD}$ vacuum as well as the relation between geometry and dynamic features of creation of the secondary particle source. The correlation measurements can be one of the focuses and significant part of the NICA-MPD physics program.

\section{References}

1. V.A. Okorokov, E.V. Sandrakova, Univ. J. Phys. Appl. 1, 196 (2013).

2. T.D. Lee, Phys. Rev. D 8, 1226 (1973); T.D. Lee, G.G.

Wick, Phys. Rev. D 9, 2291 (1974).

3. D.E. Kharzeev et al., Nucl. Phys. A 803, 227 (2008).

4. K. Fukushima et al., Phys. Rev. D 78, 074033 (2008).

5. V.A. Okorokov, Int. J. Mod. Phys. E 22, 1350041 (2013).

6. S.A. Voloshin, Phys. Rev. C 70, 057901 (2004).

7. G. Basar, D.E. Kharzeev, arXiv: 1202.2161 [hep-ph].

8. V.A. Okorokov, arXiv: 0908.2522 [nucl-th]; V.A Okorokov, Phys. At. Nucl. Eng. 4, 805 (2013).

9. V.V. Skokov et al., Int. J. Mod. Phys. A 24, 5925 (2009);

V. Voronyuk et al., Phys. Rev. C 83, 054911 (2011).

10. J. Cleymans et al., Phys. Rev. C 73, 034905 (2006).

11. M.M. Aggarwal et al., Phys. Rev. C 83, 024901 (2011).

12. L. Kumar, Nucl. Phys. A 904-905, 256c (2013).

13. V.A. Okorokov, arXiv: 1312.4269 [nucl-ex]. 2013; V.A. Okorokov, Adv. High Energy Phys. 2015, 790646 (2015).

14. V.A. Okorokov, arXiv: 1504.08336 [nucl-ex].

15. L. Valentin, Subatomic physics: nuclei and particles V. I

(Ermann, Paris, 1982); K.N. Mukhin, Experimental nuclear physics V. I (Energoatomizdat, Moscow, 1993).

16. P. Collins, An introduction to Regge theory and high energy physics (Cambridge Univ. Press, Cambridge, 1977).

17. K.A. Olive et al., Chin. Phys. C38, 090001 (2014).

18. V.A. Okorokov, Adv. High Energy Phys. 2015, 914170 (2015).

19. C. Bourrely et al., arXiv: 1202.3611 [hep-ph]. 2012.

20. E.-M. Ilgenfritz, E.V. Shuryak, Phys. Lett. B 325, 263 (1994).

21. C. Greiner, B. Müller, Phys. Lett. B 219, 199 (1989). 